\title{
Young Binary Stars in Taurus-Auriga
}

\author{
Russel J. White \\ Department of Astronomy, University of Texas at Austin, R.L.M. Hall \\ 15.308, Austin, TX 78731
}

Andrea M. Ghez

UCLA Division of Astronomy and Astrophysics, Los Angeles, CA 90095

\begin{abstract}
We present the results of a high spatial resolution ultraviolet, optical and near-infrared survey of 44 young binary stars in TaurusAuriga with separations of 10 - 1000 AU. The observations were carried out using the Hubble Space Telescope and NASA's IRTF. The binary star properties corroborate our previous work that suggests fragmentation is the dominant binary star formation mechanism. Of particular interest, we find that the components of binary systems are more coeval than randomly paired single T Tauri stars. Several important conclusions are drawn regarding the evolution of circumstellar material in binary systems. The mass accretion rates for primary stars are similar to single stars, which suggests that a companion as close as 10 AU has little effect on the mass accretion rate. These accretion rates, if constant, require replenishment of the inner circumstellar disks for at least the closest $(\lesssim 100$ AU) binary systems. On average, circumprimary disks appear to survive longer and accrete at a higher rate than circumsecondary disks do. This suggests that circumprimary disks are being preferentially replenished, possibly from a circumbinary reservoir with low angular momentum relative to the binary. The relative $\mathrm{T}$ Tauri types and the binary mass ratios tentatively suggest that systems with separations $\lesssim 200$ AU share a common circumbinary reservoir. The higher mass accretion rates of primary stars relative to secondary stars is most likely due to their larger relative mass.
\end{abstract}

\section{Introduction}

It is now well known that the majority of young solar-type stars, often called $\mathrm{T}$ Tauri stars, are in binary systems (Ghez et al. 1993; Leinert et al. 1993; Simon et al. 1995). The majority of these pairs have separations less than $100 \mathrm{AU}$, a separation that is less than the size typically assumed for a circumstellar disk. The ubiquity of these close companions has raised two important questions concerning the star formation process: "How do binary stars form?" and "How does a companion affect the distribution of circumstellar material?" Obtaining the answers to these questions requires spatially resolved measurements over a broad range of wavelengths in order to determine the stellar and circumstellar 
properties of each component separately. Unfortunately, this has been inhibited by the inherent difficulty of spatially resolving binaries with small angular separations (i.e. $\lesssim 11^{\prime \prime} 0$ ). A few studies have nevertheless had some success accomplishing this and the combined results have been useful in understanding how binary stars form (Hartigan, Strom \& Strom 1994; Brandner \& Zinnecker 1997; Ghez, White \& Simon 1997). Core fragmentation (e.g. Boss 1988) appears to be the dominant formation mechanism.

Our current understanding of the binary star formation process offers a framework with which we can begin to understand the distribution of circumstellar material in binary star systems. Numerical simulations of circumstellar material within a binary suggest that both circumprimary and circumsecondary disks are possible, as well as circumbinary structures (Artymowicz \& Lubow 1996; Bate \& Bonnell 1997). Few observational constraints are available, however, to check these predications. The combined emission from young binary stars often exhibits strong $\mathrm{H} \alpha$ emission and photospheric excesses suggesting the presence of an accreting inner circumstellar disk. However, it is in general not known whether this material is distributed in a circumprimary disk, a circumsecondary disk, or both. Ground-based spectroscopic work has identified circumprimary and circumsecondary disks in relatively wide binaries $(Z 100 \mathrm{AU}$; Brandner \& Zinnecker 1997; Prato \& Simon 1997; Duchêne et al. 1999). Studies focusing on the closer and potentially more interesting systems have been primarily limited to NIR wavelengths where high resolution techniques such as speckle imaging or adaptive optics are more realizable (e.g. Woitas, Leinert \& Köler 2000). While the components of many of these systems show signatures of retaining a circumstellar disk, details are difficult to interpret since the underlying stellar emission is unknown; the stellar properties are best extracted from optical measurements.

In order to understand the distribution and evolution of circumstellar material in young binary star systems more completely, we have carried out a high spatial resolution imaging and spectroscopic study of $44 \mathrm{~T}$ Tauri multiple star systems in the nearby star forming region Taurus-Auriga $(\mathrm{D}=140 \mathrm{pc})$. This compilation of observations represents the largest, most complete survey of binary T Tauri stars in Taurus-Auriga based on spatially resolved measurements. These data are used to determine both the stellar and circumstellar properties of the binary star components.

\section{High Spatial Resolution Imaging and Spectroscopy}

The objective of this program was to observe a complete sample of multiple $\mathrm{T}$ Tauri star systems in the Taurus-Auriga star forming region with separations ranging from $0^{\prime \prime} 07-77^{\prime \prime} 0(10-1000 \mathrm{AU})$. The possible source list, which was constructed in 1995, consists of 47 pairs in 45 systems; groups with separations less than 10 !' are considered one system (UZ Tau, UX Tau). The close binaries were identified in the high resolution multiplicity surveys of Ghez et al. (1993), Leinert et al. (1993), and Simon et al. (1995). Wide binaries were extracted from Herbig \& Bell (1988) and Hartmann et al. (1991). In this survey, binaries with separations of $10-100 \mathrm{AU}$ are referred to as close binaries and those of 100 - $1000 \mathrm{AU}$ are wide binaries. Of these 47 pairs, 46 have been observed in this 
study (DI Tau missed) and two new companions have been discovered (V410 Tau C and FW Tau C).

The observations conducted consist primarily of three sets of data: (1) high resolution NIR speckle and direct imaging measurements (NASA's IRTF) of 44 T Tauri binary systems with separations of $0{ }^{\prime} 14-7 !^{\prime \prime} 0(20-1000$ AU), (2) high resolution space-based optical and UV observations (HST WFPC2) of 17 close (10 - $100 \mathrm{AU})$, T Tauri binary systems, and (3) spatially separated low resolution (R 1500) optical spectra (HST FOS) of four close systems (GG Tau, GH Tau, V955 Tau \& XZ Tau; separation 35 - 50 AU). A detailed description of the data analysis and specific measurements are reported in White \& Ghez (2000).

\section{The Stellar Properties}

The standard method for deriving the stellar parameters relies on both a spectroscopically derived spectral type and broad-band optical photometry. The latter is measured in this study for the component stars of 25 systems based on the HST measurements of close pairs (Section 2) and ground-based measurements of the wider pairs (Hartigan et al. 1994; Kenyon \& Hartmann 1995). For systems without spatially separated spectra, the system's spectral type is assigned to the brighter component at optical wavelengths, and then converted to effective temperatures (Bessell \& Brett 1988; Luhman 1999). Line-of-sight extinctions and luminosities are then derived from the optical photometry as described in White \& Ghez (2000). To estimate the unknown spectral type of spectroscopically unresolved secondary stars, we assume the extinction to the secondary is the same as to the primary. This extinction estimate is used to deredden the observed optical color of the secondary and to estimate its spectral type and luminosity. The similar extinction assumption is supported by the extinctions derived in systems with both spatially resolved photometry and spectra (White \& Ghez 2000).

Once a stellar temperature and luminosity are assigned to both the primary and secondary stars, their masses and ages can be estimated by comparison with the predictions of PMS evolutionary models. As is discussed in White et al. (1999), the evolutionary models of Baraffe et al. (1998) are currently the most consistent with the available observational constraints. These models are adopted in the analysis presented here. The Baraffe et al. models, however, are only computed for masses between $0.025 \mathrm{M}_{\odot}$ and $1.0 \mathrm{M}_{\odot}$. In order to extract masses and ages for higher mass stars, the evolutionary models of Palla $\&$ Stahler (1999) are adopted. These models agree reasonably well at the adopted transition mass of $1.0 \mathrm{M}_{\odot}$ (White 2000). The ages derived for these binary star components range from < 1 Myrs to 19 Myrs. The stellar masses range from $0.04 \mathrm{M}_{\odot}$ to $1.47 \mathrm{M}_{\odot}$. These values are consistent with the overall population of single T Tauri stars in Taurus-Auriga (White \& Ghez 2000).

As summarized in Ghez et al. (1997) and Woitas et al. (2000), the properties of T Tauri binary stars favor fragmentation (e.g. Boss 1988) as the dominant binary star formation mechanism. The properties of the large sample of systems presented in this study also supports the fragmentation scenario. Although we do not repeat these arguments here, we highlight the remarkably coeval ages of 
binary stars and the tentative suggestion of a mass ratio dependence on binary separation.

Figure 1 displays the relative ages of binary star components versus the projected separations for the systems resolved optically. A typical difference in age can be characterized by the standard deviation of the differences in Log(age), which is 0.31 dex for these binary stars. For comparison, a sample of randomly paired single $\mathrm{T}$ Tauri stars in Taurus-Auriga is also shown in Figure 1. An ensemble of random pairings of these single stars leads to a median standard deviation of $0.54 \mathrm{dex}$, which is considerably larger than that of the binary sample (0.31 dex). T Tauri binary stars appear to be more coeval than randomly paired single $\mathrm{T}$ Tauri stars within the same star forming region.

The distribution of mass ratios shows a very weak dependence on the binary star separation. Larger mass ratios $\left(\mathrm{m}_{\mathrm{S}} / \mathrm{m}_{\mathrm{p}}\right)$ are slightly more common for the close systems than for the wide systems: $67 \%$ of close systems (10/15) have a mass ratio $>0.7$, while only $38 \%(3 / 8)$ of wide systems have mass ratios $>0.7$. A K-S test shows that there is a $90 \%$ chance that the mass ratio distributions for the relatively small sample of close and wide pairs are from a different parent population. This tentative suggestion is further investigated using the NIR flux ratios of all spatially resolved binary systems in Taurus; large flux ratios at $\mathrm{K}$ usually correspond to large mass ratios. This sample shows that wide pairs (100 - $1000 \mathrm{AU})$ appear to have larger $\mathrm{K}$ flux ratios than close pairs (10 - $100 \mathrm{AU})$. A K-S test shows that the distributions of $\mathrm{K}$ magnitude differences for close and wide pairs are different at the $91 \%$ level. Closer inspection of this sample, however, identifies and a much clearer 'break' in the distribution of $\mathrm{K}$ magnitude differences at roughly $160 \mathrm{AU}$. If the outer cutoff for "close" systems is relaxed to $160 \mathrm{AU}$, then a K-S test shows the distribution of close $(10-160 \mathrm{AU})$ and wide (160 - $1000 \mathrm{AU})$ binaries to be different at the $97 \%$ level. One limiting observational bias is that faint companions are more difficult to detect at closer separations than at wider separations. To eliminate this possible bias, a K-S test is again conducted on close and wide pairs excluding binaries with $\mathrm{K}$ magnitude differences of 3 or more ( 1 close system, 3 wide systems). The result of this comparison shows that close $(10-160 \mathrm{AU})$ pairs are still different from wider pairs $(160-1000 \mathrm{AU})$ at the $90 \%$ level, a $1.7 \sigma$ result. Although wide pairs with flux comparable to the primary star are the easiest to identify, the frequency of these pairs seems diminished relative to close binary pairs. The alternative would imply a large population of yet undetected, low mass companions at close separations, and thus an even higher binary fraction for $\mathrm{T}$ Tauri stars.

The distribution of mass ratios versus separations has important implications for models of binary star formation. Fragmentation simulations predict that only a small fraction $(\leqslant 10 \%)$ of the final stellar masses are contained in the initial protobinary fragments for systems of separation less than $\sim 100 \mathrm{AU}$ (Boss 1988; Bate 2000). For these systems the majority of the mass of the stellar components is accreted from circumbinary material (Bate \& Bonnell 1997). Consequently, Bate (2000) predict that close binary systems are more likely to have high mass ratios than wide binary systems; the closer the components are to the center of mass of the system, the more equally they will receive accreted material. The results presented here support this theory. Although the difference is only modestly significant, pairs with separations less than $\sim 160 \mathrm{AU}$ 
have a higher fraction of high mass ratio systems than wider binaries. As is explored in the following section, pairs with this separation or less also have correlated circumstellar properties and this too may be consequence of substantial circumbinary accretion.

\section{Circumstellar Properties}

Standard accretion disk diagnostics are used to characterize the accretion activity and inner circumstellar disk material associated with the components of the binary T Tauri stars studied here. Specifically, the K-L colors are used to identify warm inner disk material, the $\mathrm{EW}[\mathrm{H} \alpha] \mathrm{s}$ are used as a tracer of circumstellar accretion and the UV excesses are used to estimate mass accretion rates (Gullbring et al. 1998). Details regarding the extraction of these diagnostics are discussed in White \& Ghez (2000).

\subsection{Circumprimary and Circumsecondary Disks}

The distributions of K-L colors and mass accretion rates for the CTTS primaries are indistinguishable from the distributions for single CTTSs (White \& Ghez 2000). This result is true for both the close (10-100 AU) and wide (100-1000 AU) pairs. The similar strengths of these circumstellar emission features for primary and single CTTSs suggests that a companion star as close as $10 \mathrm{AU}$ does not disrupt the distribution of inner circumstellar material sufficiently to diminish or enhance the mass accretion rate. The circumstellar disks in the closest systems are not likely to be very massive $\left(<0.01 \mathrm{M}_{\odot}\right)$ because of tidal truncation by the companion star (Jensen et al. 1996). Given this mass upper limit, it is difficult to understand how disks have maintained the observed accretion rates $\left(\sim 10^{-8}\right.$ $\mathrm{M}_{\odot} / \mathrm{yr}$ ) for as long as the ages of the binaries. Presuming steady accretion rates, the circumstellar disks for at least the closest systems should have dissipated after $\sim 1$ Myrs, which is a few times less than the average binary star age (Section 3). One solution to this timescale problem is that the circumstellar disks are being replenished from a circumbinary reservoir. Numerical simulations have demonstrated the reality of this possibility. Although the circumprimary and circumsecondary disks are expected to be truncated by tidal effects from the companion star, the disks may in some cases be replenished episodically (Artymowicz \& Lubow 1996).

The flow of circumbinary material onto the individual circumstellar disks depends to a large extent on its angular momentum relative to the binary. If the circumbinary material has sufficient angular momentum to form a circumbinary disk, then the lower mass object is expected to be preferentially replenished; this component has closer encounters with the circumbinary reservoir (Artymowicz \& Lubow 1996). Alternatively, if the angular momentum of the surrounding material is insufficient to form a circumbinary disk, then the infalling material is expected to preferentially replenish the circumprimary disk; this material will fall toward the center of mass (Bonnell \& Bastien 1992; Bate \& Bonnell 1997). Thus if replenishment is occuring for close binaries as is suggested by their long disk lifetimes, the angular momentum of this material may be constrained by studying the relative circumstellar properties in these systems. 
Circumprimary and circumsecondary disks within the CTTS systems are compared in Figure 2 using the K-L colors and mass accretion rates. A K-L color greater than $\sim 0.3$ indicates a circumstellar accretion disk. The relative color diagram illustrates that there are no systems which support a circumsecondary disk, but no circumprimary disk. On the other hand, there are several systems with only a circumprimary disk. For systems in which both components appear to support a circumstellar disk, the primary star is usually as red or redder in K-L than the secondary. This result is true for both close and wide pairs. A K-S test shows that the distributions of K-L color differences for close and wide pairs are not significantly different. Systems with an 'infrared companion' (Koresko et al. 1997) are the primary exceptions to the finding that primary stars are redder than their companions. These components are, however, thought to be experiencing an episode of high extinction and accretion and may in fact be the more massive component.

The result that there are systems with only circumprimary disks, but no systems with only circumsecondary disks, implies that circumprimary disks are longer lived. This finding is consistent with the low angular momentum replenishment scenario. In this case, circumprimary disks will be preferentially replenished, allowing them to survive longer than circumsecondary disks.

The systematically redder K-L colors of primary stars relative to their companions suggests that circumprimary disks accrete at a higher rate (Hartigan et al. 1991). This is illustrated more directly in the diagram in Figure 2 showing the relative mass accretion rates. Primary stars have accretion rates that are comparable to or larger than that of their companion star. Since the stellar masses within each system are also known, it can also be stated that the more massive component dominates the accretion. In the bottom panels of Figure 2 , the differences in the K-L colors and mass accretion rates of primaries and secondaries are plotted versus their mass ratios. Although primaries generally have the dominant accretion signatures, the contrast between primaries and secondaries is most significant in systems with the smallest mass ratios. Since the presence of a close companion appears to have little effect on the mass accretion rate, this suggests that the higher relative accretion rates of primary stars may simply be a consequence of their higher relative mass. As shown in White \& Ghez (2000), the mass accretion rates for both CTTS binary components and single stars appear to be correlated with stellar mass. No stars with mass less than $0.5 \mathrm{M}_{\odot}$ have a mass accretion rate greater than $10^{-9} \mathrm{M}_{\odot} \mathrm{yr}^{-1}$, while the majority of higher mass stars have accretions rates above that value. Finally, the distribution of mass accretion rates for primaries and secondaries with a similar stellar mass range are indistinguishable. The higher mass accretion rates of primary stars relative to their companions can be explained due to their higher relative mass, and is not a consequence of being in a binary system.

\subsection{Co-Regulation of Circumstellar Disks}

The presence of circumstellar disks in binary systems with ages greater than the disk dissipation timescale argues strongly that the disks must be replenished. If, as seems likely, the circumstellar disks are being replenished from a common circumbinary reservoir (e.g., Bate \& Bonnell 1997), then the disk evolutionary timescales for each component within a system may be comparable. Both com- 
ponents will be replenished, with perhaps a modest preference for the primary (Section 4.1), until the circumbinary reservoir is depleted. In such a scenario, CTTS primaries should preferentially have CTTS secondaries. To investigate this, the pairing of T Tauri types is considered here. The $\mathrm{EW}[\mathrm{H} \alpha]$ is used to distinguish CTTSs and WTTSs (Martín 1998) since it is extinction independent and its spectral type dependence can easily be accounted for.

Of the 14 close pairs (2 WTTS and 12 CTTS systems) with spatially separated $\mathrm{EW}[\mathrm{H} \alpha]$ measurements, only three are mixed types (IS Tau, V807 Tau, FV $\mathrm{Tau} / \mathrm{c}$ ). Assuming a fixed distribution of primaries (2 WTTSs and 14 CTTSs) and an equal probability of taking either a WTTS or a CTTS companion (Hartmann et al. 1991), the probability to get 3 mixed systems out of 14 binaries is $\mathrm{C}_{14}^{3}(1 / 2)^{14}=2 \%$. It thus seems very unlikely that the closest pairs are evolving independently since their circumstellar properties are correlated. In contrast, for the ten wider systems (2 WTTS and 8 CTTS systems) with spatially separated $\mathrm{EW}[\mathrm{H} \alpha]$ measurements, five are mixed pairs. The circumstellar disks in wide systems appear to be evolving independently.

As suggested by Prato \& Simon (1997), a common circumbinary reservoir may be co-regulating the evolution of the binary star components. The mixed pairing of wider systems suggests an maximum binary separation of $\sim 200 \mathrm{AU}$ for co-regulation. It is worth realizing that this separation limit is comparable to the apparent break in the distribution of mass ratios at $160 \mathrm{AU}$, as inferred from $\mathrm{K}$ flux ratios (Section 3 ). Thus both the relative $\mathrm{T}$ Tauri types and the distribution of mass ratios suggest a consistent size scale for circumbinary replenishment.

\section{Summary}

We have carried out a high spatial resolution ultra-violet, optical and nearinfrared survey of 44 young binary stars in Taurus-Auriga with separations of 10 - $1000 \mathrm{AU}$. These observations are used to extract the stellar and circumstellar properties of the components of $\mathrm{T}$ Tauri binary stars systems. The stellar properties favor fragmentation as the dominant binary star formation mechanism (e.g. Boss 1988). The relative ages of binary stars are consistent with being coeval to within 1-2 Myrs. Binary stars are also shown to be more coeval than randomly paired single $T$ Tauri stars within the same star forming region. The distribution of mass ratios, as traced by NIR flux ratios, suggests that high mass ratios are more common in close pairs $(10$ - $160 \mathrm{AU})$ than in wide pairs (160 $1000 \mathrm{AU})$. This may be a consequence of significant circumbinary accretion for the closest systems (Bate 2000).

Several important conclusions are drawn regarding the evolution of circumstellar material in binary systems. The mass accretion rates for primary stars are similar to single stars, which suggests that a companion as close as $10 \mathrm{AU}$ has little effect on the mass accretion rate. These accretion rates, if constant, require replenishment of the inner circumstellar disks for the closest ( $\$ 100 \mathrm{AU}$ ) binary systems. Although there are several systems which support only a circumprimary disk, there are no systems which support only a circumsecondary disk. This suggests that, on average, circumprimary disks survive longer than circumsecondary disks do. Primary stars also appear to accrete at a higher rate than their lower mass companions do. The longer disk lifetimes of circum- 
primary disks, despite their larger accretion rates, suggests that circumprimary disks are being preferentially replenished, possibly from a circumbinary reservoir that has low angular momentum relative to the binary. The relative $\mathrm{T}$ Tauri types and the binary mass ratios also support the replenishment scenario. Their dependence on separation suggests that replenishment may be effective only in binaries with separations $\lesssim 200$ AU. The higher mass accretion rates of primary stars relative to secondary stars is most likely due to their larger relative mass. The mass accretion rates for both binary and single $\mathrm{T}$ Tauri stars appear to be mass dependent.

\section{References}

Artymowicz, P., \& Lubow, S., H. 1996, ApJ, 467, L77

Baraffe, I., et al. 1998, A\&A, 337, 403

Bate, M. R. 2000, MNRAS, 314, 33

Bate, M. R., \& Bonnell, I. A. 1997, MNRAS, 285, 33

Bessell, M., \& Brett, J. M. 1988, PASP, 100, 1134

Bonnell, I., \& Bastien, P. 1992, ApJ, 401, L31

Boss, A. P. 1988, Comments Astrophys., 12, 169

Brandner, W., \& Zinnecker, H. 1997, A\&A, 321, 220

Duchêne, G., Monin, J.-L., Bouvier, J., \& Ménard, F. 1999b, A\&A, 351, 954

Ghez, A. M., Neugebauer, G., \& Matthews, K. 1993, A\&A, 106, 2005

Ghez, A. M., White, R. J., \& Simon, M. 1997, ApJ, 490, 353

Gullbring, E., Hartmann, L. W., Briceño, C., \& Calvet, N. 1998, ApJ, 492, 323

Hartigan, P., et al. 1991, ApJ, 382, 617

Hartigan, P., Strom, K. M., \& Strom, S. E. 1994, ApJ, 427, 961

Hartmann, L., Stauffer, J. R., Kenyon, S. J., \& Jones, B. F. 1991, AJ, 101, 1050

Herbig, G. H., \& Bell, K. R. 1988, Lick Obs. Bull., 1111, 1

Jensen, E. L. N., Mathieu, R., D., \& Fuller, G., A. 1996, ApJ, 458, 312

Kenyon, S. J., \& Hartmann, L. W. 1995, ApJS, 101, 117

Koresko, C. D., Herbst, T. M., \& Leinert, Ch. 1997, ApJ, 480, 741

Leinert, Ch., et al. 1993, A\&A, 278, 129

Luhman, K. 1999, ApJ, 525, 466

Martín, E. L. 1998, AJ, 115, 351

Mathieu, R. D. 1994, ARA\&A, 32, 465

Palla, F., \& Stahler, S. W. 1999, ApJ, 525, 772

Prato, L., \& Simon, M. 1997, ApJ, 474, 455

Simon, M., et al. 1995, ApJ, 443, 625

White, R. J., \& Ghez, A. M. 2000, in prep.

White, R. J. 2000, IAU Symposium 200: The Formation of Binary Stars

White, R. J., et al. 1999, ApJ, 520, 811

Woitas, J., Leinert, Ch., \& Köhler, R. 2000, A\&A, submitted 

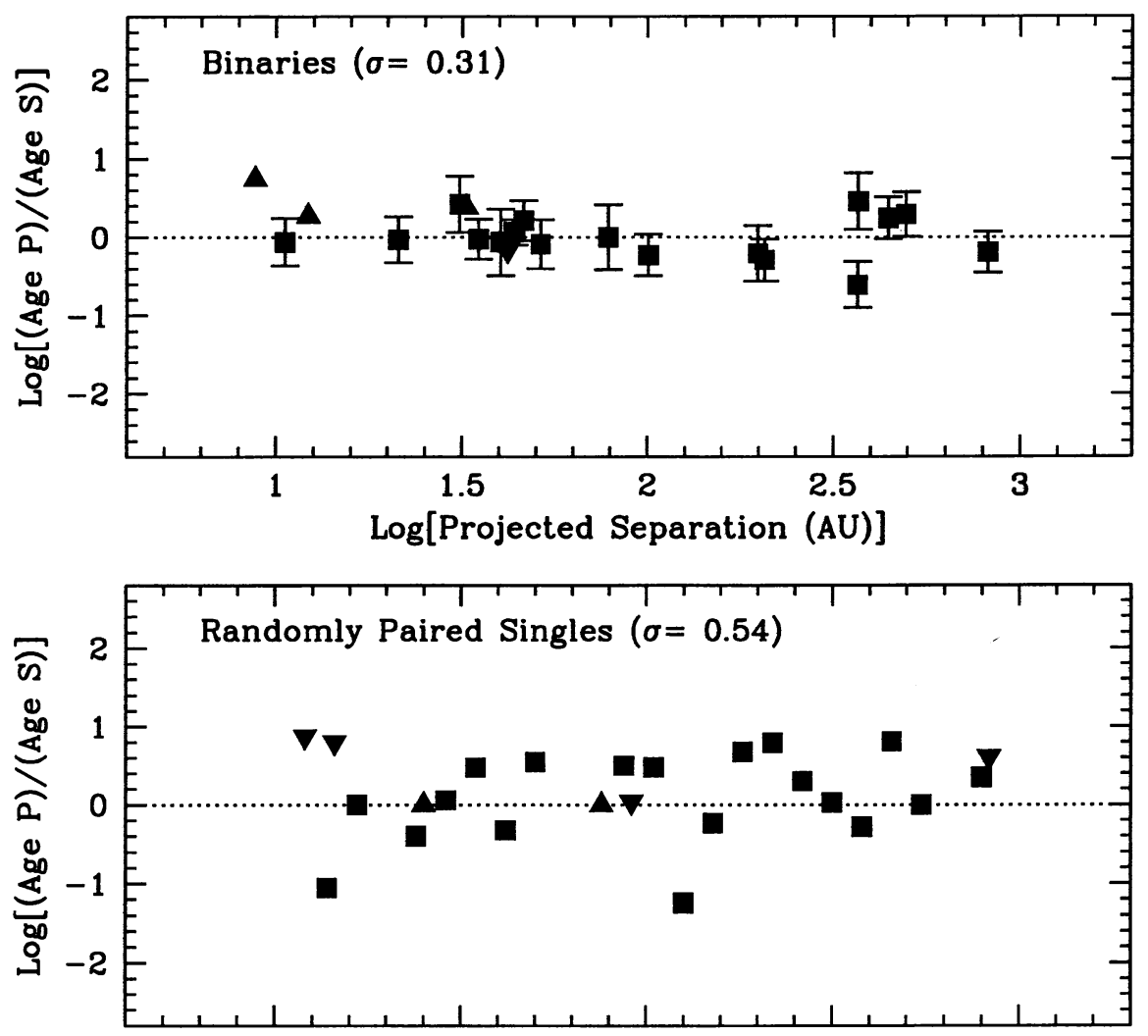

Figure 1. The relative ages of binary pairs are plotted as a function of projected separation. The triangles represent systems with either a primary age upper limit (downward pointing) or a secondary age upper limit (upward pointing). There is no statistically significant difference in age between primaries and secondaries over all separation ranges. The bottom panel shows the relative ages for a sample of randomly paired single stars, plotted with a uniform distribution of arbitrary separations. The components of binary stars are generally more coeval than isolated stars are within the same star forming region. 

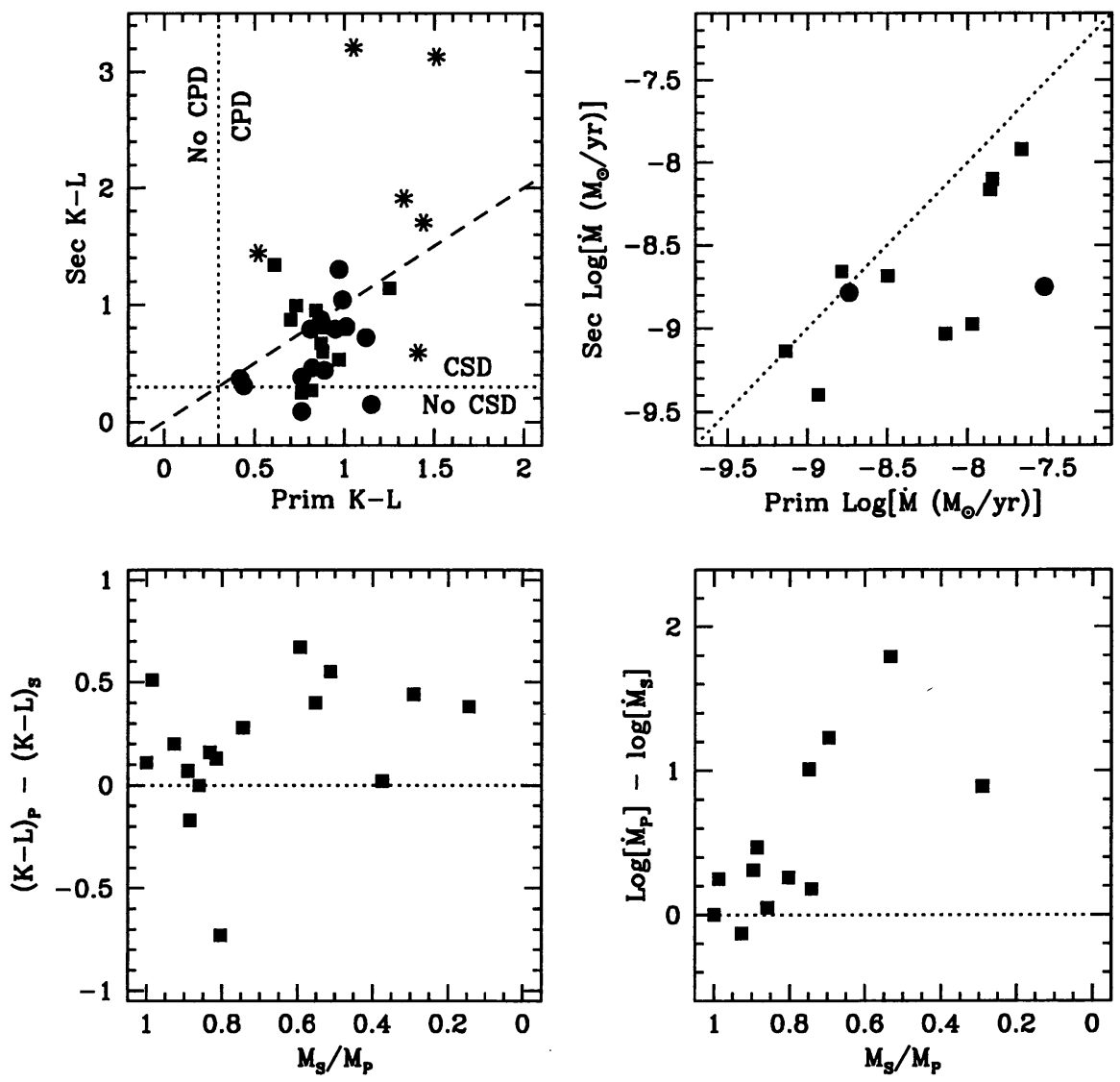

Figure 2. The K-L colors (top left panel) and mass accretion rates (top right panel) of the primaries and secondaries in CTTS systems are compared. Close pairs (10-100 AU; squares) are distinguished from wide pairs (100-1000 AU; circles). Systems with an 'infrared companion' (see Koresko et al. 1997) are plotted with an asterisk. Several systems show evidence of a circumprimary disk (CPD), but no circumsecondary disk (CSD); there are no systems with only a circumsecondary disk. Primary stars appear to have an accretion rate that is comparable to or larger than that of their companion. As shown in the bottom panels, the differences in the primaries and the secondaries accretion signatures are most significant in systems with the smallest mass ratios. 